\title{
USO DE LA ECOGRAFÍA EN LA PIELONEFRITIS AGUDA DEL ADULTO.
}

Luis García-Ferrer', Joaquín Primo², Joaquín Ulises Juan Escudero ${ }^{3}$, Felipe Ordoño Domínguez ${ }^{3}$ y José Manuel Esteban 4 .

Servicio de Radiodiagnóstico'. Consorcio Hospital General Universitario de Valencia. Valencia.

Unidad de Digestivo². Servicio de Medicina Interna. Hospital de Sagunto. Sagunto.

Servicio de Urología ${ }^{3}$. Consorcio Hospital General Universitario de Valencia. Valencia.

ERESA TC y RMㄴ. Servicio de Radiodiagnóstico. Consorcio Hospital General Universitario de Valencia. Valencia. España.

Resumen.- OBJETIVO: Evaluar la necesidad de la realización de una ecografía en los pacientes adultos afectos de una pielonefritis aguda (PNA).

MÉTODOS: Revisión de la bibliografía existente en las bases de datos PubMed y Cochrane Collaborattion sobre el uso de la ecografía para valoración y diagnóstico de PNA.

RESULTADOS: Se encontraron 37 textos de los cuales únicamente cumplian requisitos para su análisis 5 . En total se analizaron 463 pacientes de los cuales a 449 (97\%) se le realizó una ecografía por el diagnóstico de PNA. De los 171 hallazgos ecográficos, únicamente en 52 (1 1.5\%) cambiaba el diagnóstico a PNA complicada con posibilidad de tratamiento quirúrgico.
Luis García Ferrer

Servicio de Radiodiagnóstico

Consorcio Hospital General

Universitario de Valencia.

Av. Tres cruces, 2.

46014 Valencia. (España).

garcia_luifer@gva.es

Trabajo recibido: 1 de diciembre 2006.
CONCLUSION: La baja incidencia de hallazgos ecográficos no justifica su realización a todo paciente con PNA. En los pacientes con fiebre persistente más de 72 horas (h), antecedentes de anomalias de la vía urinaria, antecedentes de litiasis renal, gestación, clínica atípica o diabetes mellitus, existe una mayor incidencia de hallazgos patológicos en la ecografía que justifiquen un cambio de actitud terapéutica. Hacen falta estudios bien diseñados para confirmar estas conclusiones.

Palabras clave: Pielonefritis. Ecografía. Revisión. Indicación.

Summary.- OBJECTIVES: To evaluate the need to perform renal ultrasound (US) in adult patients with acute pyelonephritis (APN).

METHODS: A Review of the bibliography in the data bases PubMed and Cochrane Collaboration about the use of the ultrasonography in the evaluation and diagnosis of APN.

RESULTS: Thirty-seven papers were found, but only 5 fulfilled the requirements for analysis. Four hundred and sixty three patients diagnosed of APN were revised, 449 197\%) of whom got US. Between 171 ultrasonographic findings, only in $52(11.5 \%)$ cases US findings changed initial diagnosis to complicated APN that could lead to surgery.

CONCLUSION: The low incidence of ultrasonographic findings does not justify the practice of renal US to every patient with APN. In patients with persistent fever longer than 72 hours, antecedents of anomalies of the urinary tract, antecedents of renal lithiasis, pregnancy, atypical clinic or diabetes mellitus, there is a higher incidence of pathological US findings that justify a change in the the- 
rapeutic approach. Further prospective clinical studies are needed to confirm these conclusions.

Keywords: Pyelonephritis. Ultrasonography. Review. Indication.

\section{INTRODUCCIÓN}

Las infecciones del tracto urinario (ITU) son una patología frecuente que se da en todas la franjas de edad y en ambos sexos, si bien existe un claro predominio de su prevalencia en el sexo femenino (1), teniendo especial vinculación en este grupo con las relaciones sexuales, historia de ITU previas, diabetes mellitus (DM) e incontinencia urinaria (2). Esta mayor frecuencia comienza a disminuir a partir de los 60 años y se iguala aproximadamente en los 85, sobretodo por la existencia de patología prostática que favorece la existencia de un residuo vesical postmiccional con el consiguiente aumento del riesgo de infección de esta orina (3).

El diagnóstico de infección del tracto urinario conlleva la existencia de una clínica y hallazgos de laboratorio, siendo pues, un diagnóstico puramente clínico, sin necesidad de pruebas de imagen $(4,5)$. Entre las pruebas de laboratorio es indispensable la presencia de un análisis de orina con bacteriuria (patógenos en orina) en valores $>10^{4.5} \mathrm{UFC} / \mathrm{ml}$, a la cual se puede asociar leucocitosis. El urocultivo con resultado positivo mostrará en aproximadamente un $90 \%$ el aislamiento de E.Coli (1).

Las ITU podemos clasificarlas en función de si afectan a vías bajas (cistitis-uretritis) o altas (pielonefritis) $(4,5)$. Los síntomas de una infección de vías bajas consisten en disuria, polaquiuria, urgencia miccional y dolor suprapúbico. Para el diagnóstico de infecciones de vías altas, es necesaria la presencia de fiebre mayor de $38^{\circ}$, dolor continuo en flanco, posibilidad de escalofríos, náuseas y vómitos junto a síntomas de vías bajas (6).

Si bien ambas son patologías frecuentes, es la pielonefritis aguda (PNA) el cuadro que requiere una mayor atención por su posibilidad de desembocar en un absceso renal o shock séptico, con aumento de la gravedad clínica $(7,8)$. Por ello, y a pesar que en su mayoría, con el tratamiento médico adecuado curan rápidamente, se ha intentado identificar el grupo de pacientes afectos de PNA que tienen un mayor riesgo de presentar una mala evolución clínica (9-17). Entre los medios utilizados para la evaluación de la PNA están las pruebas de imagen, y más concretamente la ecografía. El objetivo principal de la realización de esta prueba es valorar la existencia de una dilatación de vía urinaria por obstáculo $u$ otra complicación que obligue a un cambio en actitud terapéutica con uso de manipulación instrumental (9). Sin embargo, no existe ninguna pauta establecida para la indicación de esta prueba de imagen, existiendo múltiples guías, que en ocasiones llegan a dar recomendaciones enfrentadas en función de a qué sociedad pertenecen $(8,18,19)$. Siguiendo en esta línea, todavía hay menos textos que traten el uso de la ecografía de urgencia en la valoración de la PNA.

Nos hemos propuesto hacer una revisión de la literatura con el objetivo de establecer en que situaciones la realización de una ecografía renal en una PNA está indicada.

\section{MATERIAL Y MÉTODO}

Se ha realizado una búsqueda bibliográfica en la Cochrane Collaboration y en Medline usando los términos MESH "pyelonephritis", "ultrasonography", "diagnosis" y "guidelines" en distintas combinaciones asociando los artículos referenciados en la bibliografía, en los vínculos de Medline "artículos relacionados" así como revisiones y libros de texto. Sólo se pudieron revisar los textos escritos en inglés o español.

Los abstracts de todos los artículos fueron leídos y se consiguieron aquellos textos que a priori parecían cumplir los requisitos definidos para esta revisión: artículos de series de pacientes con diagnóstico clínico de PNA a los que se les realizó una ecografía para evaluar la presencia de alteraciones renales. Los autores leyeron los textos que cumplían estos requisitos, siendo rechazados aquellos que no aportaban información suficiente para el análisis de datos, no definían bien el grupo de estudio o se trataban de revisiones del tema.

\section{RESULTADOS}

Se han encontrado 37 textos. De ellos, únicamente 12 eran estudios de pruebas de diagnóstico por la imagen. Tras el análisis de los trabajos, se descartaron 3: dos debido a que la población de estudio no eran pacientes diagnosticados de PNA $(14 ; 20)$ y uno porque el estudio se centra en otras pruebas diagnósticas (17).

De los 9 estudios restantes, en uno no se explica el tipo de estudio, 6 son retrospectivos y 2 prospectivos. Centrándonos en los hallazgos ecográficos, no se pudieron obtener los datos en 4 trabajos: Kanel (10) compara el uso de la urografía intravenosa 
(UIV) y ecografía en pacientes sin factores de riesgo realizando la exploración última sólo a un $40 \%$ y sin especificar los hallazgos obtenidos por prueba diagnóstica. Shen (13) estudia a 66 pacientes en los que la prueba de imagen podía ser ecografía, TAC, UIV, Rx de abdomen o una combinación de ellas. Sólo en un $56 \%$ disponemos de ecografía y sin especificar los hallazgos por prueba. Force (15), analiza a 91 pacientes en un estudio prospectivo, de los cuales un $96 \%$ tienen ecografía y el $34 \%$ UIV. Si bien sólo hay hallazgos en un $13 \%$ de los pacientes, no especifica los mismos por estudio de imagen. Finalmente, Rollino (21) escoge a 52 pacientes en función de su diagnóstico de alta por clasificación CIE-9, el estudio por imagen fue a elección del clínico: ecografía, TAC, medicina nuclear, cistouretrografías y nuevamente en los hallazgos se centra por patología sin especificar que técnica dio el diagnóstico.

Tras la exclusión de los 7 trabajos citados anteriormente, quedan para su análisis 5 estudios $(1,9,11,12,16)$. En todos ellos se valoraron pacientes diagnosticados de PNA aunque existen variaciones en la definición de la patología y en la selección de la población (Tabla I). El número de pacientes estudiados es de 463, realizándose ecografía dentro de las primeras $72 \mathrm{~h}$ a 449 de ellos (97\%). No se ha podido hallar la edad media de la población al expresarse ésta en media +/- desviación esntándar o mediana en función del artículo. Sólo en 3 trabajos se incluyeron tanto hombres como mujeres, siendo la distribución de 266 (79\%) mujeres y 72 (21\%) hombres.

De las ecografías realizadas, hubo hallazgos en 171 (38\%) (Tabla II). No hay datos sobre los resultados en función del sexo. Hemos clasificado la patología encontrada (Tabla III) en función de si modificaba o no la actitud terapéutica de manera urgente (pielonefritis no complicada-pielonefritis complicada) con el resultado de una disminución de dichos hallazgos al $11 \%$.

Basándose en los antecedentes personales, Neal (1) encuentra una relación estadísticamente significativa entre hallazgos ecográficos significativos y la diabetes mellitus (DM). Roca-Tey (12) únicamente objetiva una mayor estancia media y más tiempo en quedar afebril.

\section{DISCUSIÓN}

La justificación de solicitar una prueba de imagen en el estudio de la PNA es descartar la presencia de alteraciones anatómicas, signos de obstrucción (con o sin litiasis) o abscesos renales (18). Entre las alteraciones anatómicas, destaca por su frecuencia la existencia de una duplicación del sistema pielocalicial. Se trata de la anomalía congénita más frecuente de la vía urinaria, con una incidencia del $0.5-10 \%$ (22) en la que el polo superior e inferior drenan a través de sistemas diferentes que pueden ser independientes cada uno con su propio uréter hasta vejiga (duplicación completa) o unirse en el trayecto y drenar en un uréter único (duplicación incompleta). Independientemente, esta malformación predispone al reflujo y a la posibilidad de obstrucción de la unión pieloureteral. La obstrucción del tracto urinario por una litiasis conlleva un aumento del riesgo de infección de la orina retenida que ecográficamente se representa por presencia de ecos en el interior del líquido y que llamamos pionefrosis. El absceso renal tiene la apariencia ecográfica de una lesión redondeada, hipoecoica o anecoica, con ecos en su interior y rodeada por paredes gruesas (7). Tanto el absceso renal como la pionefrosis son indicaciones de actitud invasiva.

TABLA I. CRITERIOS DE DEFINICIÓN DE PIELONEFRITIS AGUDA EN LOS ARTÍCULOS SELECCIONADOS.

\begin{tabular}{|llllllll|}
\hline & Fiebre & $\begin{array}{l}\text { Sedimento } \\
\text { patológico }\end{array}$ & $\begin{array}{l}\text { Clínica } \\
\text { miccional }\end{array}$ & $\begin{array}{l}\text { Urinocultivo + Dolor en } \\
\text { flanco }\end{array}$ & Leucocitosis Exclusión de pacientes \\
con factores de riesgo
\end{tabular}

* Roca-Tey define PNA como clínica compatible (sin especificar signos o síntomas), leucocitosis y sedimento urinario patológico. 
Los hallazgos descritos en las ecografías en pacientes con pielonefritis aguda podemos dividirlos en dos grupos:

a) Hallazgos casuales (que no interfieren en la patología o en el tratamiento).

b) Hallazgos patológicos (que su existencia puede influir en la evolución o requerir cambio de tratamiento). Consideramos hallazgos patológicos la uropatía obstructiva, alteraciones de la vía urinaria, presencia de abscesos renales y la pielonefritis enfisematosa $(7,23)$. Tanto el aumento del tamaño renal, como el edema renal y la rarefacción de la grasa perirrenal son descritas en los textos radiológicos $(7,11,24)$ como imágenes sugestivas de pielonefritis que no implican aumento de la gravedad. De hecho, dichas imágenes no se consideraron en varios textos como alteraciones, dado que se presuponía su posible visualización durante la realización de la ecografía.

TABLA II. HALLAZGOS ECOGRÁFICOS DENTRO DE LAS PRIMERAS 72H EN PACIENTES INGRESADOS POR PIELONEFRITIS AGUDA.

\begin{tabular}{|lll|}
\hline Hallazgos ecográficos & N & $\%$ \\
Hidronefrosis & 10 & 6 \\
Absceso perinefrítico & 3 & 2 \\
Pielonefritis enfisematosa & 1 & 0.6 \\
Litiasis con obstrucción & 13 & 8 \\
Linfocele & 1 & 0.6 \\
Litiasis renal sin hidronefrosis & 35 & 21 \\
Pielonefritis crónica & 12 & 7 \\
Aumento del tamaño renal & 45 & 26 \\
Necrosis papilar & 1 & 0.5 \\
Riñón poliquístico & 2 & 1 \\
Ectasia intrarrenal & 21 & 12 \\
Absceso renal & 5 & 3 \\
Nefritis focal aguda & 4 & 2 \\
Riñón único & 2 & 1 \\
Alteración de la vía urinaria & 14 & 8 \\
Riñón en esponja & 1 & 0.6 \\
Uropatía obstructiva no filiada & 1 & 0.6 \\
TOTAL & 171 & 100 \\
\hline
\end{tabular}

En la revisión actual, el porcentaje de hallazgos ecográficos se sitúa en el $38 \%$, disminuyendo a un $11 \%$ si consideramos los hallazgos sugestivos de PNA complicada, y que es realmente la justificación de la realización de una prueba de imagen.

A tener en cuenta también el uso de varios términos descriptivos en función de los hallazgos ecográficos en la infección renal. Términos como nefritis bacteriana aguda, nefritis bacteriana focal, nefritis lobar, nefronía lobar, preabsceso, celulitis renal y flemón renal se encuentran a lo largo de la literatura, ya sea en estudios por imagen con urografía intravenosa, ecografía o TAC. Tal abundancia de nombres puede conllevar a la confusión del clínico, y es por ello que en 1994 (25) la sociedad de urorradiología promoviese la nomenclatura única de pielonefritis aguda que se acompaña de la descripción: focal o difusa, con edema o aumento del tamaño renal, unilateral o bilateral y complicada (presencia de absceso, obstrucción o signos de aire) o no. De esta manera, el hallazgo del aumento del tamaño renal se describe como pielonefritis aguda con aumento del tamaño renal, y la existencia de una región con disminución de la ecogenicidad pero sin apariencia líquida ni cápsula, se cita como pielonefritis aguda focal.

Siguiendo con la nomenclatura y clasificación de esta patología, podemos distinguir entre dos tipos: la PNA no complicada y PNA complicada (1). La diferencia radica en la existencia de hallazgos patológicos entendidos tal y como se ha descrito anteriormente. Puesto que en un $89 \%$ de las infecciones no hay alteraciones significativas en la prueba de imagen, la pregunta que se plantea es si realmente hay una indicación de realizar ecografía a todos los pacientes atendidos por PNA. En este respecto, hay disensión entre clínicos y radiólogos: la asociación europea de urología publicó en 2001 (8) y recientemente ha actualizado (26) las guías en infecciones de orina y tracto genital masculino en las cuales se recomienda, con grado de evidencia $C(27 ; 28)$, la realización de una ecografía en el caso de PNA no complicada en ambos sexos. Siguiendo esta guía, artículos de revisión publicados en revistas de urología (29), continúan la recomendación. Sin embargo, si uno de basa en las guías oficiales de radiología $(18,19)$, la indicación de ecografía queda reservada a aquellos pacientes con un aumento del riesgo de presentar una PNA complicada: diabéticos, inmunocomprometidos, historia de litiasis, clínica de cólico renal asociado, falta de respuesta a una adecuada antibioterapia en $72 \mathrm{~h}$ y antecedentes de infecciones urinarias de repetición. Este último punto, es controvertido, ya que si bien la sospecha es la existencia de una alteración anatómica o funcional que facilite la repetición de ITU, cuando se realiza un estudio de 
TABLA III. CLASIFICACIÓN DE LOS HALLAZGOS ECOGRÁFICOS EN FUNCIÓN DE SU CAPACIDAD DE MODIFICAR LA ACTITUD TERAPÉUTICA.

\begin{tabular}{|l|l|l|l|}
\hline \multicolumn{2}{|c|}{ PIELONEFRITIS AGUDA NO COMPLICADA } & \multicolumn{2}{c|}{ PIELONEFRITIS AGUDA COMPLCADA } \\
\hline & $\mathbf{N}(\%)$ & Hidronefrosis & 10 (6) \\
\hline Litiasis renal sin hidronefrosis & $35(21)$ & Absceso perinefrítico & $3(2)$ \\
\hline Pielonefritis crónica & $12(7)$ & Pielonefrituis enfisematosa & $1(0.6)$ \\
\hline Aumento del tamaño renal & $45(26)$ & Litiasis con obstrucciòn & $13(8)$ \\
\hline Riñón poliquístico & $2(1)$ & Linfocele* & $1(0.6)$ \\
\hline Ectasia intrarrenal & $21(12)$ & Absceso renal & $5(3)$ \\
\hline Necrosis papilar & $1(0.6)$ & Uropatía obstructiva no filiada & $1(0.6)$ \\
\hline Riñón en esponja & $1(0.6)$ & Alteración de la vía urinaria & $14(8)$ \\
\hline Riñón único & $2(1)$ & & \\
\hline Nefritis focal aguda & $4(2)$ & TOTAL & $\mathbf{4 8}(\mathbf{2 8})$ \\
\hline TOTAL & $\mathbf{1 2 3 ( 7 2 )}$ & & \\
\hline
\end{tabular}

* Hallazgo etiquetado como PNA complicada(1)

imagen, éste es en su gran mayoría normal (30). Por último, la lectura de artículos de revisión $(31,32)$, sugiere como indicaciones los antecedentes de riesgo (ya comentados) de pacientes y coinciden en la no necesidad de un estudio por imagen en la PNA no complicada en la mujeres. En las embarazadas, se acepta que debe hacerse siempre una ecografía. El motivo no es otro que la gravedad potencial y la trascendencia de las complicaciones en la embarazada. En el sexo masculino, dada la mayor prevalencia de alteraciones de la vía urinaria en pacientes con ITU(3), no se cuestiona el uso de la ecografía.

De los artículos revisados, destaca por su claridad el estudio realizado por Ortega (16). Estableciendo a priori dos grupos de pacientes con PNA en función de la existencia o no de factores de riesgo para la presencia de PNA complicada (fiebre que persiste más de $72 \mathrm{~h}$ a pesar de antibioterapia, clínica atípica, gestación, antecedentes de litiasis y anomalías en la vía urinaria), obtuvo una sensibilidad del $62 \%$ para hallazgos ecográficos. No hemos encontrado ningún artículo que confirme esos resultados, siendo los últimos publicados en la literatura meramente descriptivos.

Hay que destacar la baja proporción de hallazgos significativos en nuestra revisión. En varios trabajos ésta ronda el 20-25\%, no obstante, si bien la definición de PNA es aceptable en los artículos revisados, en dos de ellos fue criterio de exclusión el tener factores de riesgo para tener una PNA complicada, por lo que nuestros resultados están afectados por un sesgo de población de estudio.

\section{CONCLUSIONES}

A pesar de ello, y coincidiendo con otros autores $(1,23,24,30,32-34)$ pensamos que la realización de una ecografía debe limitarse a pacientes con PNA y factores de riesgo para PNA complicada. A estos factores, hay que añadir la DM, asociada a alteraciones de la inmunidad, problemas de vaciamiento de vejiga y a la posibilidad de una lesión renal subyacente. Son necesarios más estudios correctamente dirigidos para detectar la población con factores de riesgo para presentar una PNA complicada ya que este grupo se beneficiaría de una actuación más dirigida.

\section{BIBLIOGRAFIA y LECTURAS RECOMENDADAS ( ${ }^{*}$ lectura de interés $y^{* *}$ lectura fundamental)}

**1. NEAL, D.E.; STEELE, R.; SLOANE, B.: "Ultrasonography in the differentiation of complicated and uncomplicated acute pyelonephritis". Am. J. Kidney. Dis., 16: 478, 1990.

2. SCHOLES, D.; HOOTON, T.M.; ROBERTS, P.L. y cols.: "Risk factors associated with acute pyelonephritis in healthy women". Ann. Intern. Med., 142: $20,2005$. 
**3. LIPSKY, B.A.: "Urinary tract infections in men. Epidemiology, pathophysiology, diagnosis, and treatment". Ann. Intern. Med., 110: 138, 1989.

4. MANDELL, G.L.; BENNETT, J.E.; DOLIN, R.: "Principles and practice of infectious diseases". 6th ed. Philadelphia: Elsevier-Churgchill-Livingstone, 875, 2005.

5. KASPER, D.L.; BRAUNWALD, E.; FAUCI, A.S. y cols.: "Harrison's Principles of internal medicine". 16th ed. McGraw-Hill, 1715, 2005.

6. RAMAKRISHNAN, K.; SCHEID, D.C.: "Diagnosis and management of acute pyelonephritis in adults". Am. Fam. Physician., 71: 933, 2005.

7. PAPANICOLAOU, N.; PFISTER, R.C.: "Acute renal infections”. Radiol. Clin. North Am., 34: 965, 1996.

8. NABER, K.G.; BERGMAN, B.; BISHOP, M.C. y cols.: "EAU guidelines for the management of urinary and male genital tract infections. Urinary Tract Infection (UTI). Working Group of the Health Care Office (HCO) of the European Association of Urology (EAU)". Eur. Urol., 40: 576, 2001.

9. LUJAN GALAN, M.; PAEZ BORDA, A.; FERNANDEZ GONZALEZ, I. y cols.: "Utilidad de la ecográfica en la evaluación de la pielonefritis aguda”. Arch. Esp. Urol., 50: 46, 1997.

10. KANEL, K.T.; KROBOTH, F.J.; SCHWENTKER, K. y cols.: "The intravenous pyelogram in acute pyelonephritis". Arch. Intern. Med., 148: 2144, 1988.

11. JOHNSON, J.R.; VINCENT, L.M.; WANG, K. y cols.: "Renal ultrasonographic correlates of acute pyelonephritis". Clin. Infect. Dis., 14: 15, 1992.

12. ROCA-TEY, R.; GARCIA-OSUNA, R.; TORGUET, P. y cols.: "Pielonefritis aguda. Estudio de 153 casos". Nefrologia, 20: 373, 2000.

13. SHEN, Y.; BROWN, M.A.: "Renal imaging in pyelonephritis". Nephrology, 9: 22, 2004.

14. WANG, I.K.; CHANG, F.R.; YANG, B. y cols.: "The use of ultrasonography in evaluating adults with febrile urinary tract infection". Ren. Fail., 25: 981, 2003.

15. FORCE, L.; MURGUI, L.; BARRUFET, P. y cols.: "Pielonefritis aguda. Análisis prospectivo de 91 casos". Rev. Clin. Esp., 188: 223, 1991.

**16. ORTEGA ENCISO, L.; SANCHEZ MARTINEZ, F.; ESCAPE DIAZ-BONILLA, I. y cols.: "Indicaciones clínicas de la ecográfica en la pielonefritis aguda en mujeres adultas". Rev. Clin. Esp., 198: 647, 1998.

17. SATTARI, A.; KAMPOURIDIS, S.; DAMRY, N. y cols.: "CT and 99mTc-DMSA scintigraphy in adult acute pyelonephritis: a comparative study". J. Comput. Assist. Tomogr., 24: 600, 2000.

18. SANDLER, C.M.; AMIS, E.S.; BIGONGIARI, L.R. y cols.: "Imaging in acute pyelonephritis. American College of Radiology. ACR Appropriateness Criteria”. Radiology, 215: 677, 2000.
19. MARTÍ BONMATÍ, L.; BOUZAS, R.; BRU, y cols. "Criterios de remisión de pacientes a los servicios de radiología. Sociedad Española de Diagnóstico por la Imagen de Abdomen (SEDIA)". Sociedad Española de Radiología Médica (SERAM). http:// www.seram.es/formacion_criterios_revision_sedia.php 2002.

20. JUNE, C.H.; BROWNING, M.D.; SMITH, L.P. y cols.: "Ultrasonography and computed tomography in severe urinary tract infection". Arch. Intern. Med. 145: 841, 1985.

21. ROLLINO, C.; BOERO, R.; FERRO, M. y cols.: "Acute pyelonephritis: analysis of 52 cases". Ren. Fail., 24: 601, 2002.

22. RUMACK, C.; WILSON, S.; CHARBONEAU, J.: "Diagnóstico por ecografía". Marban, 329, 1999.

*23. KAWASHIMA, A.; LEROY, A.J.: "Radiologic evaluation of patients with renal infections". Infect. Dis. Clin. North Am., 17: 433, 2003.

24. NOBLE, V.E.; BROWN, D.F.: "Renal ultrasound". Emerg. Med. Clin. North Am., 22: 641, 2004.

**25. TALNER, L.B.; DAVIDSON, A.J.; LEBOWITZ, R.L. y cols.: "Acute pyelonephritis: can we agree on terminology?". Radiology, 192: 297, 1994.

*26. NABER, K.G.; BERGMAN, B.; BISHOP, M.C. y cols.: "EAU guidelines for the management of urinary and male genital tract infections. Urinary Tract Infection (UTI) Working Group of the Health Care Office (HCO) of the European Association of Urology (EAU)". Update, 2006.

27. WEIDNER, W.; LUDWIG, M.; WEIMAR, B. y cols.: "Rational diagnostic steps in acute pyelonephritis with special reference to ultrasonography and computed tomography scan". Int. J. Antimicrob. Agents, 11: 257, 1999.

28. ENGEL, J.D.; SCHAEFFER, A.J.: "Evaluation of and antimicrobial therapy for recurrent urinary tract infections in women". Urol. Clin. North Am., 25: $685,1998$.

29. BJERKLUND JOHANSEN, T.E.: "Diagnosis and imaging in urinary tract infections". Curr. Opin. Urol., 12: 39, 2002.

30. STAMM, W.E.; HOOTON, T.M.: "Management of urinary tract infections in adults". N. Engl. J. Med., 329: 1328, 1993.

31. HOOTON, T.M.; STAMM, W.E.: "Indications for radiologic evaluation in acute pyelonephritis". Up To Date, Vol 13 No2. http://www.utdol.com/utd/ content/topic.do

*32. FIHN, S.D.: "Clinical practice. Acute uncomplicated urinary tract infection in women". N. Engl. J. Med., 349: 259, 2003.

33. KAWASHIMA, A.; SANDLER, C.M.; GOLDMAN, S.M.: "Current roles and controversies in the imaging evaluation of acute renal infection". World J. Urol., 16: 9, 1998.

34. HOOTON, T.M.; STAMM, W.E.: "Diagnosis and treatment of uncomplicated urinary tract infection". Infect Dis. Clin.. North Am., 11: 551, 1997. 